\title{
Flexible Pressure-Sensitive Contact Transistors Operating in Subthreshold Regime
}

\author{
Sanghoon Baek ${ }^{1, \ddagger}$, Geun Yeol Bae $e^{2,3, \ddagger}$, Jimin Kwon ${ }^{1}$, Kilwon Cho ${ }^{2, *}$, Sungjune Jung ${ }^{1,2, *}$
}

${ }^{1}$ Department of Creative IT Engineering, Pohang University of Science and Technology (POSTECH), 77 Cheongam-Ro, Nam-Gu, Pohang, 37673, Republic of Korea

${ }^{2}$ Department of Chemical Engineering, Pohang University of Science and Technology (POSTECH), 77 Cheongam-Ro, Nam-Gu, Pohang, 37673, Republic of Korea

${ }^{3}$ Intelligent Sustainable Materials R\&D Group, Research Institute of Sustainable Manufacturing System, Korea Institute of Industrial Technology, Cheonan-si 331-822, Chungcheongnam-do, Republic of Korea

* Corresponding Author: kwcho@postech.ac.kr, sjjung@postech.ac.kr

\section{Contents}

Figure S1. Device characteristics of the staggered organic TFT .....................................2

Figure S2. Microscopic images of the contact area .........................................................

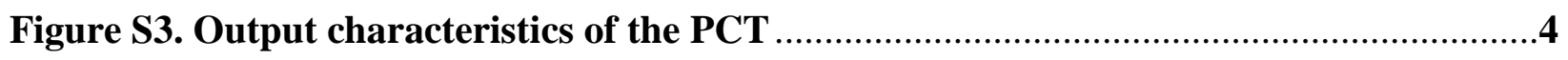

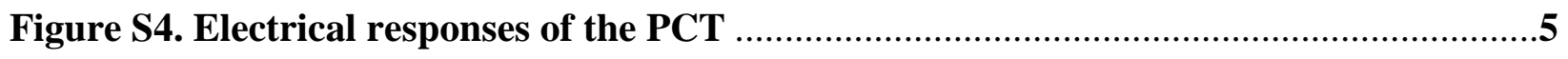

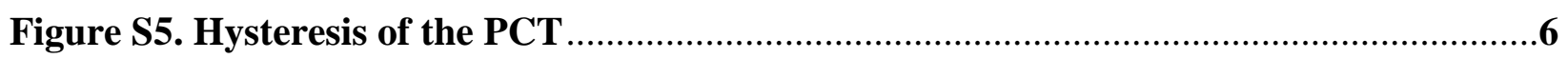

Figure S6. Design of the active matrix PCT array ......................................................

Table S1. Summary of previously reported organic TFT-based E-skins ............................8 
(a)

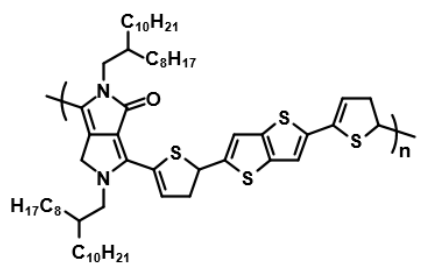

(d)

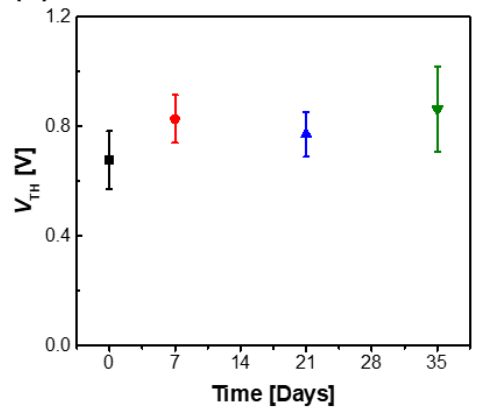

(b)

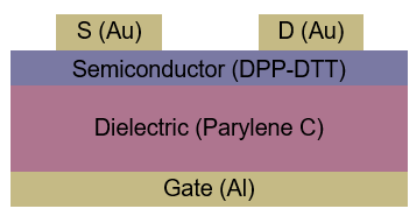

(e)

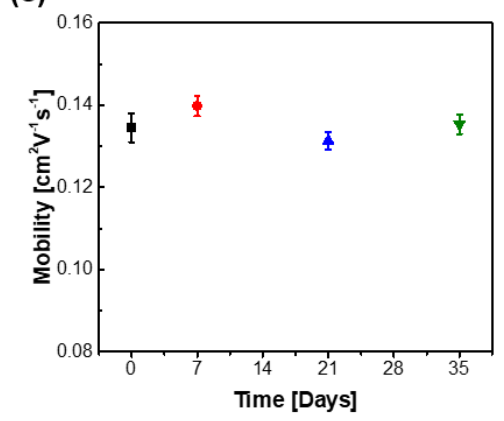

(c)

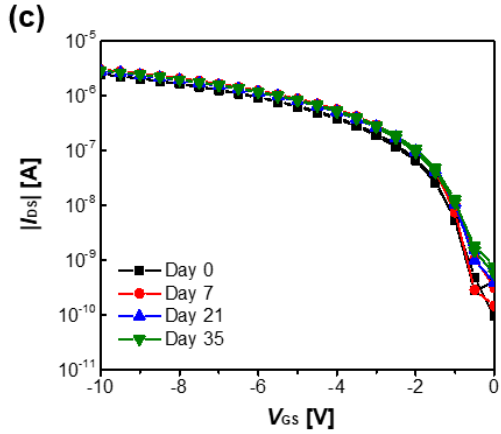

(f)

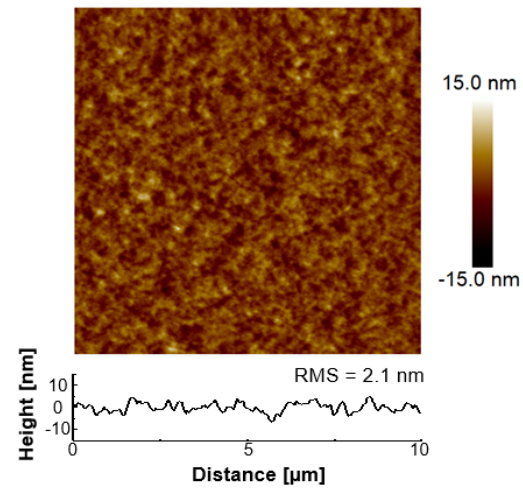

Figure S1. Device characteristics of the staggered organic TFT (with vapor deposited metal S/D electrodes). (a) Chemical structure of Poly(N-alkyl diketopyrrolo-pyrrole dithienylthieno[3,2b] thiophene) (DPP-DTT). (b) Schematic diagram of the staggered organic TFT (with vapordeposited metal S/D electrodes). (c) Transfer characteristics of the staggered organic TFT measured for $35 \mathrm{~d}\left(V_{\mathrm{DS}}=-10 \mathrm{~V}\right)$. (d) Corresponding threshold voltage $\left(V_{\mathrm{TH}}\right)$ shift for $35 \mathrm{~d}$ extracted from (c). (e) Corresponding change in carrier mobility for $35 \mathrm{~d}$ extracted from (B). (f) AFM image of the surface of the semiconductor (DPP-DTT) with a root mean square (RMS) roughness $=2.1$ nm. Bars: \pm 1 s.d., $\mathrm{n}=3$. 

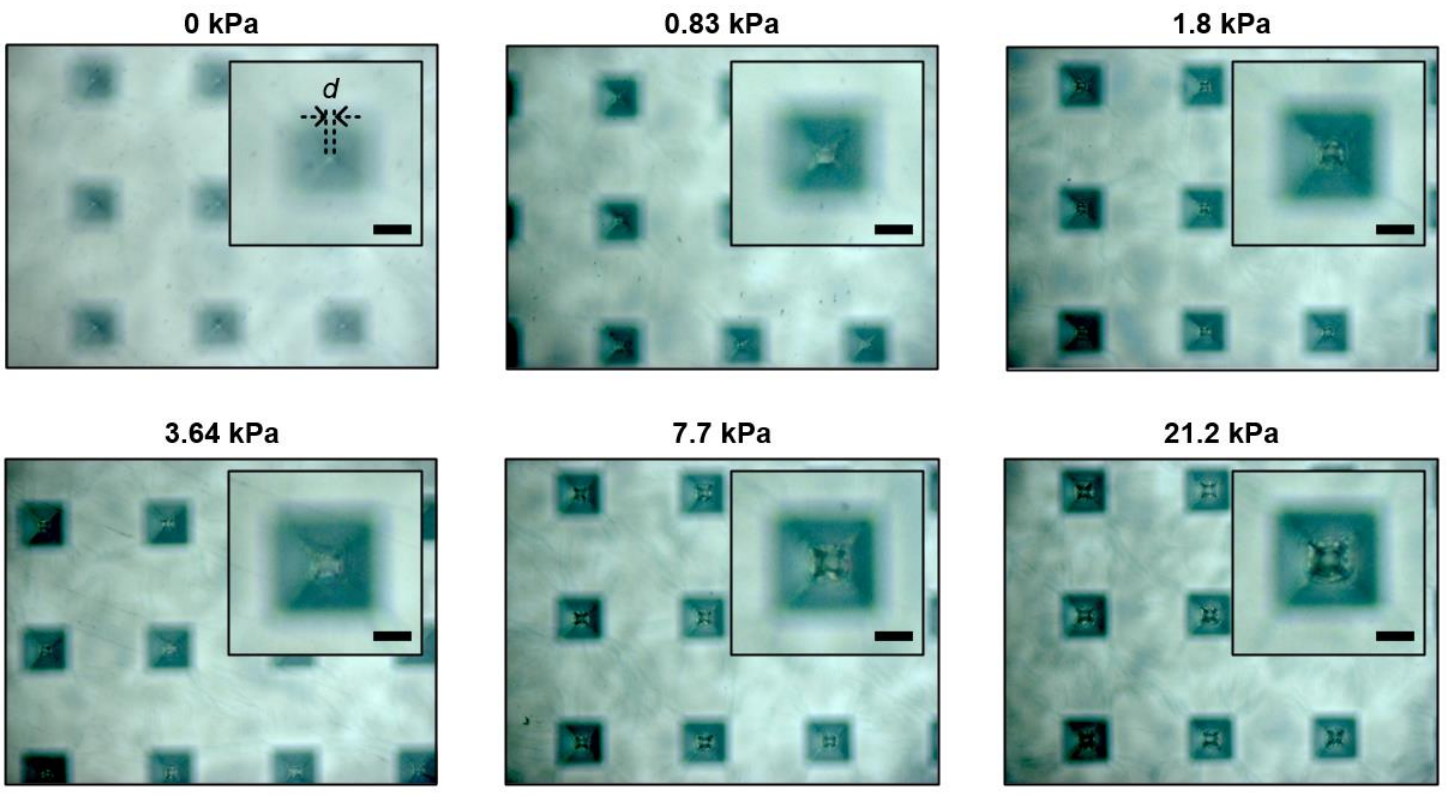

Figure S2. Microscopic images of the contact area between the deformable S/D electrodes and semiconductor upon applied pressure. Insets: magnified images of single pyramids (Scale bar $=$ $10 \mu \mathrm{m})$. 

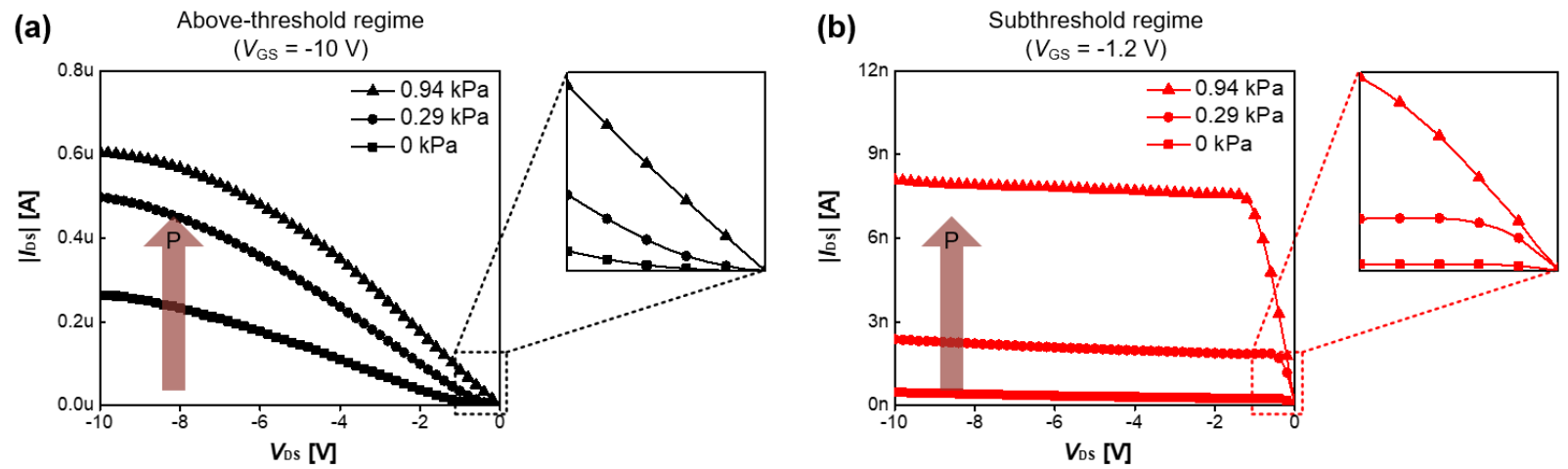

Figure S3. Output characteristics of the PCT upon applied pressure at two different regimes. a) above-threshold regime $\left(V_{\mathrm{GS}}=-10 \mathrm{~V}\right)$, and b) subthreshold regime $\left(V_{\mathrm{GS}}=-1.2 \mathrm{~V}\right)$. Insets: magnified graphs where $V_{\mathrm{DS}}<1 \mathrm{~V}$. 
(a)

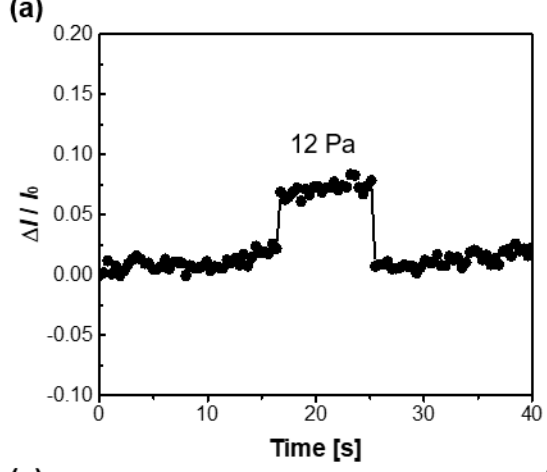

(c)

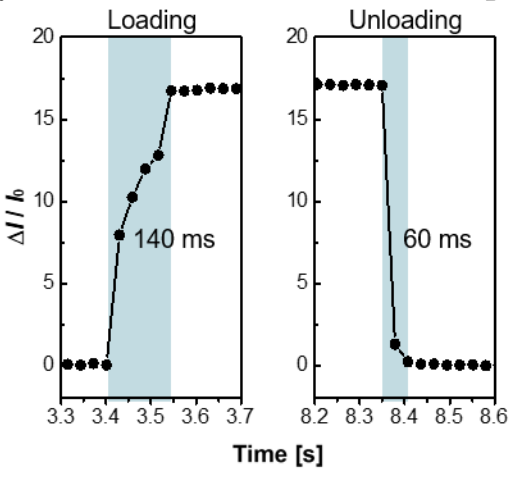

(b)

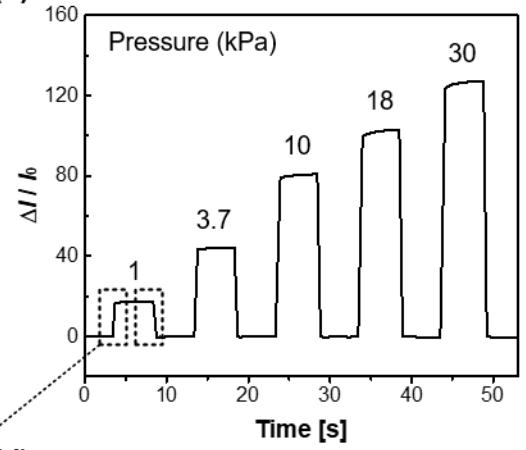

(d)

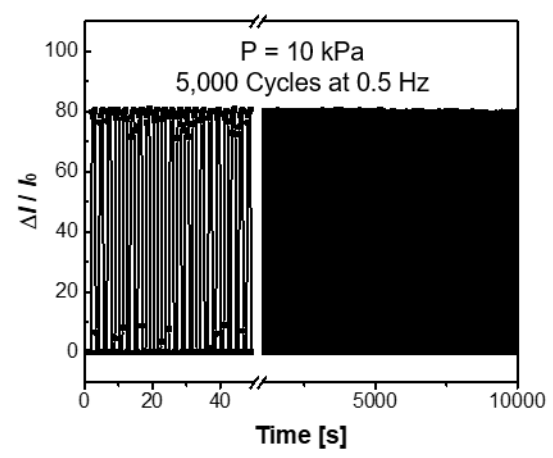

Figure S4. Electrical responses of the PCT to the applied pressure. (a) Limit of detection (LOD) of the PCT (12 Pa). (b) Representative response of the PCT to gradually increasing pressure loads. (c) Response time of the PCT when $1 \mathrm{kPa}$ is applied. (d) Durability of the PCT under a pressure of $10 \mathrm{kPa}$ for 5,000 cycles. All electrical responses were measured in the subthreshold regime ( $V_{\mathrm{GS}}$ $=-1.2 \mathrm{~V})$. 


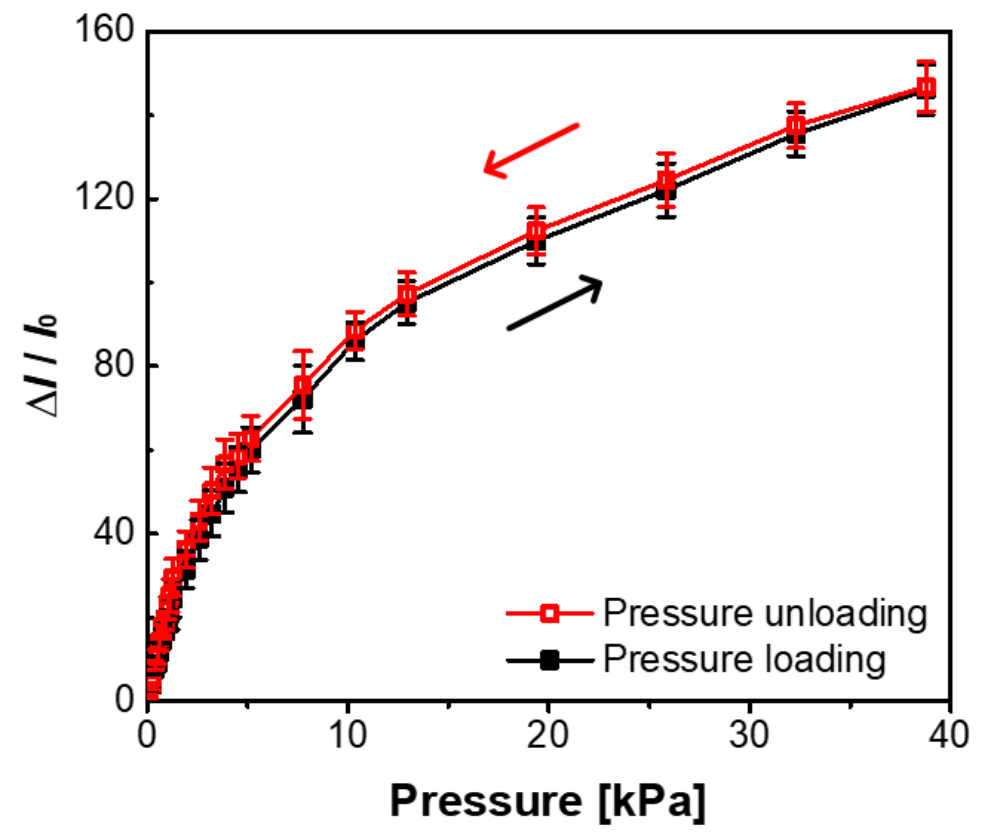

Figure S5. Hysteresis of the PCT by measuring the drain current under loading/unloading pressure. Bars: \pm 1 s.d., $\mathrm{n}=3$. 
(a)

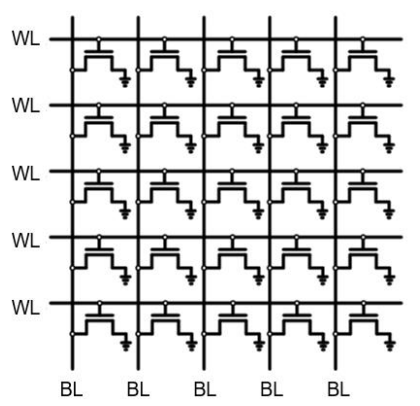

(b)

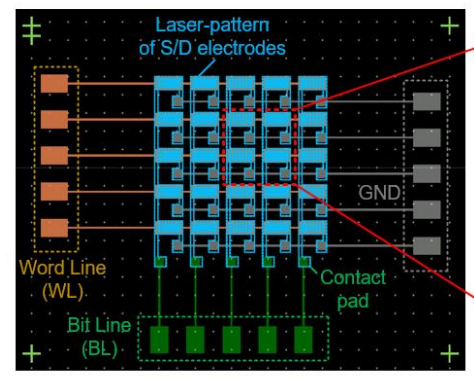

(c)

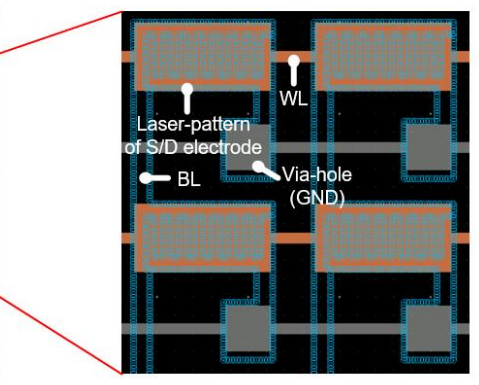

Figure S6. Design of the active matrix PCT array. (a) Circuit diagram of the $5 \times 5$ active matrix PCT array (WL: word line, and BL: bit line). (b) Design layout of the PCT array. (c) Magnified image of the design layout of each cell of the PCT array. 
Table S1. Summary of previously reported organic TFT-based E-skins.

\begin{tabular}{|c|c|c|c|c|c|c|}
\hline $\begin{array}{l}\text { Transducing } \\
\text { Mechanism }\end{array}$ & Integration & Operating voltage & $\begin{array}{l}\text { Operating } \\
\text { regime }\end{array}$ & $\begin{array}{c}\text { Operating } \\
\text { power level }[\mu \mathrm{W}]\end{array}$ & $\begin{array}{c}\text { Pressure } \\
\text { sensitivity }\left[\mathrm{kPa}^{-1}\right] \\
\end{array}$ & Ref. \\
\hline \multirow{11}{*}{$\begin{array}{l}\text { One transistor } \\
\text { - one resistor } \\
\quad(\Delta R)\end{array}$} & \multirow{9}{*}{ Pressure-sensitive rubber } & $V_{G S}=-20 \mathrm{~V} / V_{D S}=-20 \mathrm{~V}$ & Above-T & 10 & $\mathrm{n} / \mathrm{a}$ & [10] \\
\hline & & $V_{G S}=-20 \mathrm{~V} / V_{D S}=-20 \mathrm{~V}$ & Above-T & 10 & $\mathrm{n} / \mathrm{a}$ & [15] \\
\hline & & $V_{G S}=-40 \mathrm{~V} / V_{D S}=-40 \mathrm{~V}$ & Above-T & 1 & $\mathrm{n} / \mathrm{a}$ & [46] \\
\hline & & $V_{\mathrm{GS}}=-5 \mathrm{~V} / V_{\mathrm{DS}}=-3 \mathrm{~V}$ & Above-T & 100 & $11.5 \mu \mathrm{S}$ & [11] \\
\hline & & $V_{G S}=-5 V / V_{D S}=-1 \mathrm{~V}$ & Above-T & 100 & $30 \mu \mathrm{S}$ & [16] \\
\hline & & $V_{\mathrm{GS}}=-5 / V_{\mathrm{DS}}=10 \mathrm{~V}$ & Above-T & 10000 & $42.7 \mathrm{Cdm}^{2}$ & [17] \\
\hline & & $V_{G S}=-10 \mathrm{~V} / V_{D S}=-5 \mathrm{~V}$ & Above-T & 1 & 7 & [19] \\
\hline & & $V_{G S}=-3 V / V_{D S}=-1 V$ & Above-T & 1 & $\mathrm{n} / \mathrm{a}$ & [47] \\
\hline & & $V_{G S}=-3 V / V_{D S}=-3 V$ & Above-T & 100 & $\mathrm{n} / \mathrm{a}$ & [48] \\
\hline & Pressure-sensitive graphene & $V_{G S}=-2 \mathrm{~V} / V_{D S}=-0.3 \mathrm{~V}$ & Above-T & 0.1 & 0.12 & [18] \\
\hline & Pressure-sensitive nanofiber & $V_{G S}=-10 \mathrm{~V} / V_{D S}=-10 \mathrm{~V}$ & Above-T & 100 & $\mathrm{n} / \mathrm{a}$ & [20] \\
\hline \multirow{11}{*}{$\begin{array}{l}\text { Deformable dielectric } \\
\qquad(\Delta C)\end{array}$} & \multirow[b]{2}{*}{ Microstructured PDMS } & $V_{\mathrm{GS}}=-80 \mathrm{~V} / V_{\mathrm{DS}}=-80 \mathrm{~V}$ & Above-T & 10 & 0.55 & [22] \\
\hline & & $V_{G S}=-100 \mathrm{~V} / V_{D S}=-100 \mathrm{~V}$ & $\begin{array}{l}\text { Above-T } \\
\text { / Sub-T }\end{array}$ & 1000 & 8.4 & [12] \\
\hline & PDMS & $V_{G S}=-80 \mathrm{~V} / V_{D S}=-80 \mathrm{~V}$ & Above-T & 10 & 1.07 & [43] \\
\hline & Microstructured P(VDF-TrFE) & $V_{G S}=-20 \mathrm{~V} / V_{D S}=-20 \mathrm{~V}$ & Above-T & 0.1 & 1.016 & [24] \\
\hline & Polyurethane nano-needle & $V_{\mathrm{GS}}=-50 \mathrm{~V} / V_{\mathrm{DS}}=-10 \mathrm{~V}$ & Above-T & 100 & 1.76 & [42] \\
\hline & \multirow{6}{*}{ Air gap } & $V_{G S}=-20 \mathrm{~V} / V_{D S}=-20 \mathrm{~V}$ & Above-T & 0.01 & 0.4 & [25] \\
\hline & & $V_{G S}=-5 V / V_{D S}=-5 V$ & Above-T & 1 & 56.15 & \multirow{2}{*}{ [44] } \\
\hline & & $V_{G S}=-2 V / V_{D S}=-2 V$ & Above-T & 1 & 8.25 & \\
\hline & & $V_{G S}=-60 \mathrm{~V} / V_{D S}=-60 \mathrm{~V}$ & Above-T & 100 & 192 & [120 \\
\hline & & $V_{G S}=-6 \mathrm{~V} / V_{D S}=-6 \mathrm{~V}$ & Above-T & 0.01 & 0.23 & \\
\hline & & $V_{G S}=25 \mathrm{~V} / V_{D S}=0.1 \mathrm{~V}$ & Above-T & 1 & 0.000205 & [23] \\
\hline \multirow{3}{*}{$\begin{array}{l}\text { Piezoelectric TFT } \\
(\text { (P(VDF-TrFE }))\end{array}$} & Piezoelectric dual gate & $V_{\mathrm{GS}}=-1.8 \mathrm{~V} / V_{\mathrm{DS}}=-5 \mathrm{~V}$ & Sub-T & 0.01 & 0.52 & [26] \\
\hline & \multirow{2}{*}{ Piezoelectric capacitor } & $V_{G S}=-2 V / V_{D S}=-2 V$ & Above-T & 1 & $<0.002^{\text {a) }}$ & [21] \\
\hline & & $V_{G S}=-2 V / V_{D S}=-2 V$ & Above-T & 1 & $<0.0001^{\text {a) }}$ & [14] \\
\hline Triobotronic TFT & Tribotronic contact pad (PTFE) & $V_{\mathrm{DS}}=2 \mathrm{~V}$ & - & 100000 & $\mathrm{n} / \mathrm{a}$ & [49] \\
\hline PCT (This work) & Deformable S/D electrodes & $V_{\mathrm{GS}}=-1.2 \mathrm{~V} / V_{\mathrm{DS}}=-10 \mathrm{~V}$ & Sub-T & 0.01 & 18.96 & \\
\hline
\end{tabular}

a) calculated by the authors from the experimental data found in literature. 